\title{
Granulocyte-like myeloid derived suppressor cells (G-MDSC) are increased in multiple myeloma and are driven by dysfunctional mesenchymal stem cells (MSC)
}

\author{
Cesarina Giallongo ${ }^{1,2, *}$, Daniele Tibullo, ${ }^{1,3, *}$, Nunziatina L. Parrinello ${ }^{1}$, Piera La \\ Cava $^{1}$, Michelino Di Rosa ${ }^{2}$, Vincenzo Bramanti ${ }^{2}$, Cosimo Di Raimondo ${ }^{1}$, Concetta \\ Conticello ${ }^{1}$, Annalisa Chiarenza ${ }^{1}$, Giuseppe A. Palumbo ${ }^{1}$, Roberto Avola ${ }^{2}$, Alessandra \\ Romano $^{1, *}$, Francesco Di Raimondo ${ }^{1, *}$ \\ ${ }^{1}$ Division of Hematology, A.O.U. Policlinico-OVE, Catania, University of Catania, Italy \\ ${ }^{2}$ Department of Biomedical and Biotechnological Sciences, University of Catania, Catania, Italy \\ ${ }^{3}$ Department of Biological, Geological and Environmental Sciences, University of Catania, Catania, Italy \\ *These authors contributed equally to this work \\ Correspondence to: Daniele Tibullo, email: d.tibullo@unict.it
}

Keywords: MM microenvironemnt, mesenchymal stem cells, G-MDSC, immune-suppression

Received: October 30, 2015

Accepted: February 05, 2016

Published: March 07, 2016

\section{ABSTRACT}

Granulocytic-Myeloid-derived suppressor cells (G-MDSC) are increased in Multiple Myeloma (MM) patients but the mechanisms of G-MDSC generation are still unknown. There are many evidences of the role of mesenchymal stem cells (MSC) in promoting MM cell growth, survival and drug-resistance. We here used a specific experimental model in vitro to evaluate the ability of MSC to induce G-MDSC. We found that although MSC derived from healthy donors (HD), MGUS and MM were able to generate the same amount of MDSC, only MM-MSC-educated G-MDSC exhibited suppressive ability. In addition, in comparison with MSC derived from HD, MM-MSC produce higher amount of immune-modulatory factors that could be involved in MDSC induction. Compared to G-MDSC obtained from co-culture models with MSC from healthy subjects, both MGUS and MM-MSC-educated G-MDSC showed increase of immune-modulatory factors. However, only MM-MSC educated G-MDSC 1) up-regulated immune-suppressive factors as ARG1 and TNFa, 2) expressed higher levels of PROK2, important in angiogenesis and inflammatory process, and 3) showed ability to digest bone matrix.

Our data demonstrate that MM-MSC are functionally different from healthy subjects and MGUS-MSC, supporting an evolving concept regarding the contribution of MM-MSC to tumor development and progression.

\section{INTRODUCTION}

Monoclonal gammopathies encompasse a spectrum of clinical variants ranging from monoclonal gammopathy of uncertain significance (MGUS) through smoldering Multiple Myeloma (MM), up to the most aggressive, symptomatic MM and plasma cell leukemia $[1,2]$. There are several evidences indicating that development of MM is due not only to uncontrolled proliferation of plasma cells $(\mathrm{PC})$ but also to change in the bone marrow (BM) microenvironment [3]. Here PC are hosted in special niches and receive multiple signals that maintain their long survival and exert a protective effect on druginduced apoptosis, due to the secretion of soluble factors, such as IL6 and extracellular vescicles [4-6]. Within the microenvironment, the host immune system has a pivotal role for the PC growth, proliferation, survival, migration and resistance to drugs and is responsible for some clinical manifestations of MM [4, 7, 8].

Myeloid-derived suppressor cells (MDSC) include myeloid cells at different stage of maturation characterized by the ability to suppress immune responses, including $\mathrm{T}$ 
cell proliferation and cytokine production [9-11]. Several groups highlighted the emerging role of MDSC in MM pathogenesis and clinical behavior, and have documented their increase in both peripheral blood (PB) and BM of MM patients [4, 12-18].

Based on the expression of surface antigens and studies available in mice, two main subpopulations of MDSC can be distinguished: $\mathrm{CD}_{11 \mathrm{~b}^{+}} \mathrm{CD}^{-}$

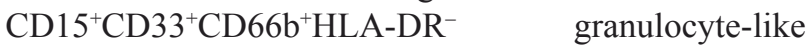
(G-MDSC) and CD14 ${ }^{+} \mathrm{CD} 15^{-} \mathrm{HLA}^{-\mathrm{DR}^{-}}$monocytelike (Mo-MDSC) [5, 19-21]. MDSC are able to inhibit the immune system by multiple mechanism, mostly through production of arginase 1 (ARG1), nitric oxidase synthase 2 (NOS2), reactive species of oxygen (ROS), cyclooxygenase 2 (COX2), transforming growth factor $\beta$ (TGF- $\beta$ ) and immunosuppressive cytokines, such as IL6, IL10 and IL1 $\beta$ [22]. In addition, MDSC can induce regulatory T-cells [22] and differentiate in functional osteoclasts contributing to the formation of osteolytic lesions in solid tumors [23-25].

BM mesenchymal stem cells (MSC) are essential components in the formation and function of the BM microenvironment [26-28]. They are a heterogeneous population of stromal adult stem cells with an important role into the tumor microenvironment due to their immunosuppressive ability, such as the capacity to inhibit $\mathrm{T}$ cell activation and proliferation. Indeed, MSC can interfere with the recognition of tumor cells by immune system producing and releasing immunoregulatory factors as TGF $\beta$, prostaglandin E2 (PGE2), tumor necrosis factor $\alpha(\mathrm{TNF} \alpha)$, indolamine 2, 3-dioxygenase (IDO), hemeoxygenase (HO), NOS2, $\mathrm{ARG}_{1-2}$, IL10 [29-32]. MSC express programmed death ligand 1 (PD-L1) that after its engagement with PD-1 expressed on T lymphocytes lead to the inhibition of $\mathrm{T}$ cell activation and proliferation with an inefficient immune response [29, 33].

Even though we and others have demonstrated increased levels of MDSC in PB from MM patients and characterized their immunosuppressive role [4, 12-16], the role of MSC in MDSC expansion and activation into the BM microenvironment remains unexplored.

In the present study, we investigated the role of MSC obtained from MGUS and MM patients on expansion and activation of G-MDSC compared to MSC from healthy donors (HD).

\section{RESULTS}

\section{Increased frequency of G-MDSC in MM patients}

The frequency of G-MDSC $\left(\mathrm{CD} 11 \mathrm{~b}^{+} \mathrm{CD} 15^{+} \mathrm{CD} 14^{-}\right.$ $\mathrm{HLADR}^{-}$cells) was evaluated in the $\mathrm{PB}$ of $\mathrm{HD}$, MGUS and $\mathrm{MM}$ patients at diagnosis and relapsed using flow cytometry. Percentage of G-MDSC was significantly higher in $\mathrm{PB}$ of patients with newly diagnosed $(65.1 \pm 11.9 \%)$, and relapsed $(80.1 \pm 10.2 \%) \mathrm{MM}$ compared to MGUS $(54.7 \pm 6.3)$ and healthy subjects $(58.2 \pm 4.6 \%)(p=0.03$ and $p<0.001$ respectively $)$ (Figure 1A).

We next evaluated the immune-suppressive activity of MDSC. For this purpose, we incubated G-MDSC obtained from MM and HD with autologous CFSE-labeled $\mathrm{T}$ cells and we found that only MM G-MDSC were able to reduce autologous $\mathrm{T}$ cells proliferation $(44.3 \pm 2.3 \% \mathrm{vs}$ $30.0 \pm 1.5 \%, p=0.009$ ) (Figure 1B).

\section{MM-MSC promote induction of MDSC in the microenvironment of MM patients}

We next assessed the role of tumor microenvironment on expansion and activation of MDSC, focusing our attention on MSC.

To explore their influence in the induction of MDSC, PBMC from healthy donors were co-cultured with HD-, MGUS- or MM-MSC for one week. Then, the frequency of G-MDSC in PBMC was analyzed before cell magnetic separation. Both HD-, MGUS- and MMMSC accumulated similar small amount of G-MDSC (Supplementary Figure 1). Next, we analyzed immunesuppressive activity of MSC-educated G-MDSC (MSCedG-MDSC). These cells were obtained with magnetic cell separation and the G-MDSC phenotype was confirmed by cytofluorimetric analysis (Supplementary Figure 2). Interestingly, we found that only MM-MSCed-G-MDSC were able to suppress T-cells proliferation $(p=0.001)$, while this suppressive activity was not recorded for MGUS-MSCed-G-MDSC or HD-MSCed-G-MDSC or G-MDSC control (isolated from PBMC cultured in medium alone) (Figure 2).

Therefore, even if HD-, MGUS- and MM-MSC are able to generate similar amount of G-MDSC cells, only MM-MSCed-G-MDCS exhibited suppressive effect on T cell proliferation.

\section{Molecular regulators of MM MSC-mediated MDSC activation}

In many cancers, it has been demonstrated that tumor-associated microenvironment produces a large amount of immune-modulating factors involved in reprogramming immature myeloid cells to become immunosuppressive G-MDSC and to attract them at the tumor sites [36]. These immunomodulatory factors include PTGS2, TGF $\beta$, NOS2, IL10, TNF $\alpha$, IL1 $\beta$, and IL6. Therefore, we first analyzed their expression by MM-MSC compared to HD-MSC at Time 0. A great variability of expression was observed among the patients, but no up-regulation of the genes above described was observed (Figure 3A). After $48 \mathrm{~h}$ of co-culture with PBMC, MM-MSC showed statistically significant up-regulation of PTGS2 (5.8 $\pm 5, p=0.018), \operatorname{TGF} \beta(27.8 \pm 34, p=0.03)$, NOS2 $(20 \pm 25.8, p=0.04), \operatorname{IL} 10(19 \pm 1, p=0.017)$ 
and IL6 $(40.7 \pm 22, p=0.02)$ expression compared with HD-MSC (Figure 3B), suggesting that MM-MSC are functionally different from HD-MSC and are able to produce higher amount of immunomodulatory factors that could be involved in MDSC generation.

\section{Gene expression changes in MM MSC-educated G-MDSC}

Before incubation with $\mathrm{T}$ cells, the relative expression levels of our set of immune modulatory factors was also investigated in MM- and MGUS-MSCed-GMDSC compared to HD-MSCed-G-MDSC. With the exception of TGF $\beta$, all the other immune-modulatory factors were found up-regulated in both MGUS- and MM-MSCed-G-MDSC, although up-regulation of TNF $\alpha$ $(45.7 \pm 28.8, p=0.002)$ was almost exclusive of MM MSCed-G-MDSC (Figure 4).

We also found that only MM-MSCed-G-MDSC significantly upregulated PROK2 $(6.4 \pm 1.7, p=0.03)$ and

\section{A}

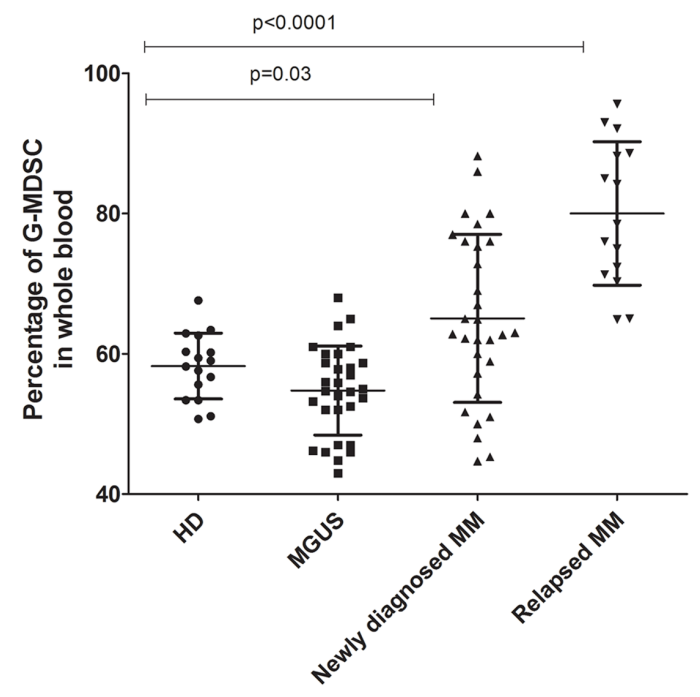

ARG1 $(111.5 \pm 50.2, p=0.001)$. PROK2 is a molecule involved in angiogenesis and inflammatory process $[37,38]$, while ARG1 is an enzyme with a key role in immunosuppression. Releasing ARG1, myeloid cells deplete L-arginine and profoundly suppress $\mathrm{T}$ cell immune response [39].

\section{MM-MSC-educated G-MDSC can digest bone matrix}

Since MM patients have an enhanced bone resorption and increased inflammatory signals into the microenvironment, we next investigated if MM-MSCeducated G-MDSC may be involved in the digestive process of bone. Therefore, G-MDSC control, HD-, MGUS- and MM-MSCed-G-MDSC were plated onto dentine disks (DDs) for 3 days. A significant digestive activity was observed only in DDs with MM-MSCed-G$\operatorname{MDSC}(p=0.002)$ (Figure 5).

B
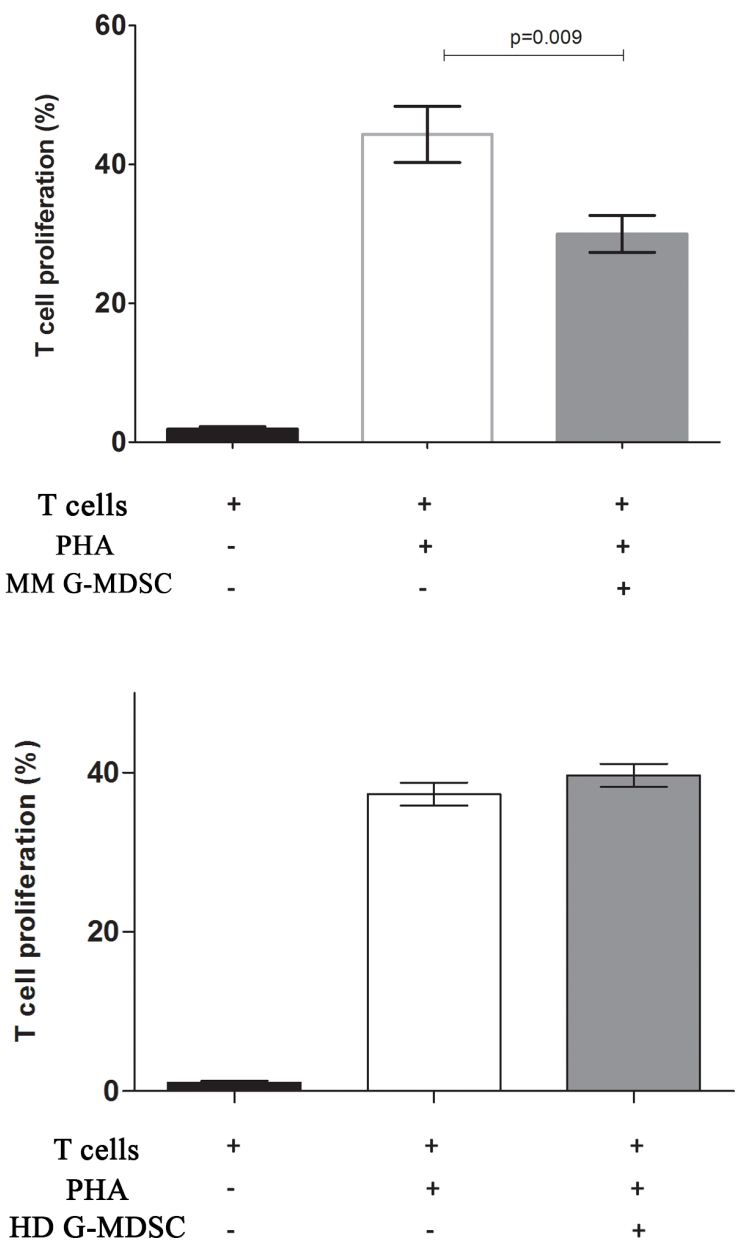

Figure 1: Increased frequency of G-MDSC in MM patients. The percentage of circulating G-MDSC was quantified in the peripheral blood of healthy donors, MGUS and newly diagnosed or relapsed MM patients by flow cytometry (panel A). MM G-MDSCmediated T-cells suppression was assessed in autologous co-cultures. Mean frequency of CD3+CFSE dim cells \pm SD from four independent experiments in duplicate is shown (panel B). 
A
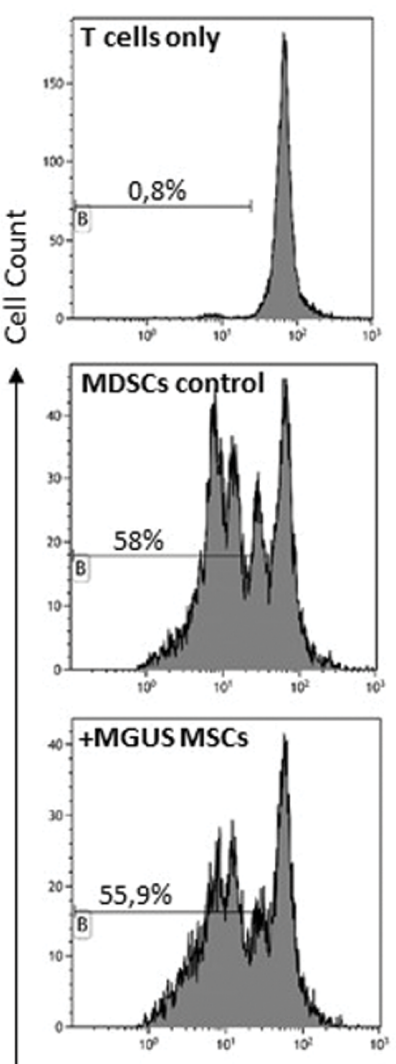
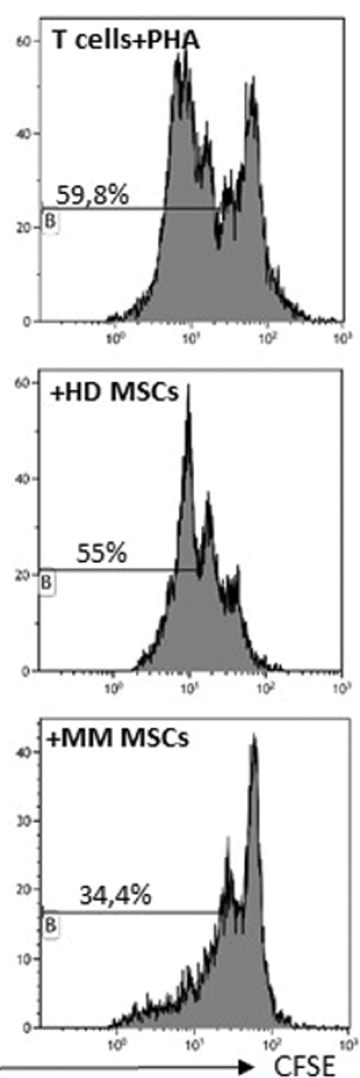

B

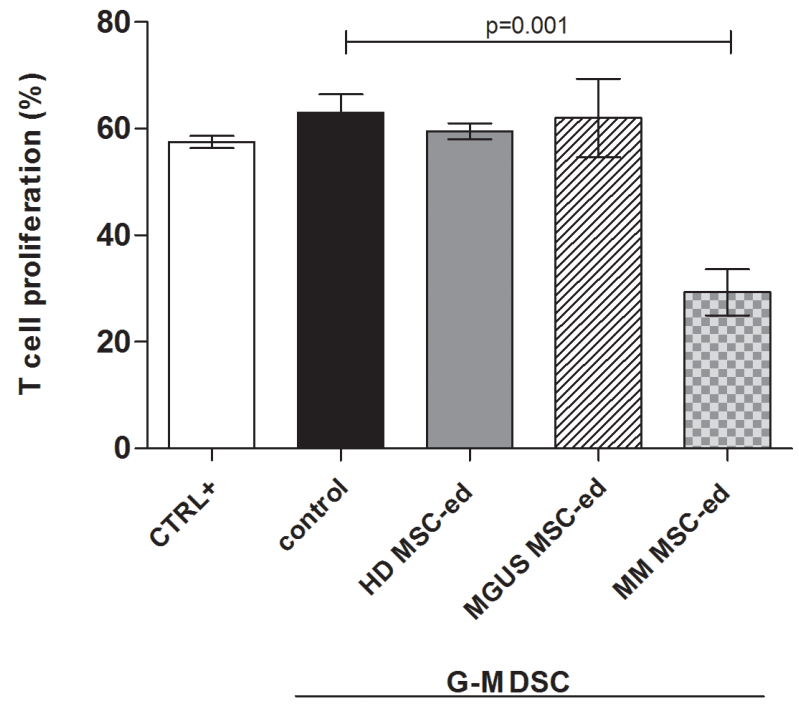

Figure 2: MM MSC educated G-MDSC are immunosuppressive. MSCed-G-MDSC were analyzed for their immune-suppressive activity against autologous T-cells. Representative flow cytometry dot-plots show the gating strategy for each experimental condition (panel (A). Only MM MSCed-G-MDSC exhibited suppressive effects compared to G-MDSC control $(p<0.001$, panel (B). The data represent mean $\pm \mathrm{SD}$ of all analyzed co-cultures in triplicate.

A

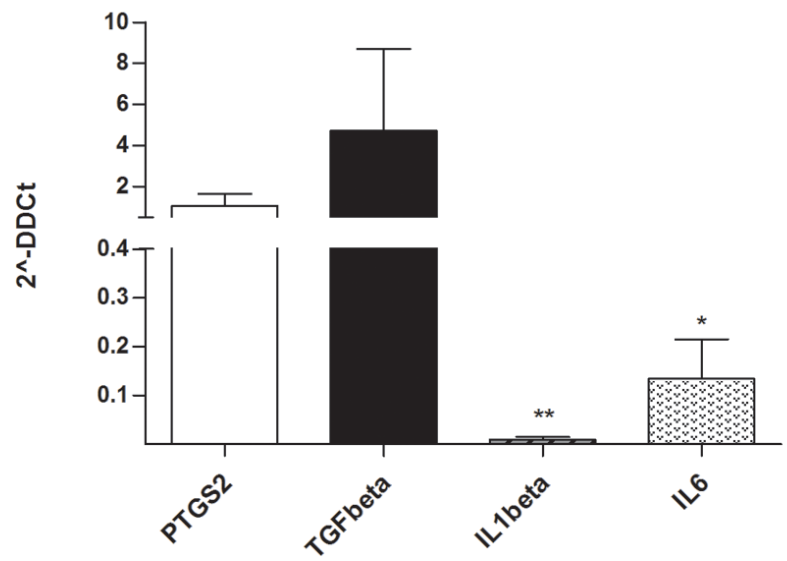

B

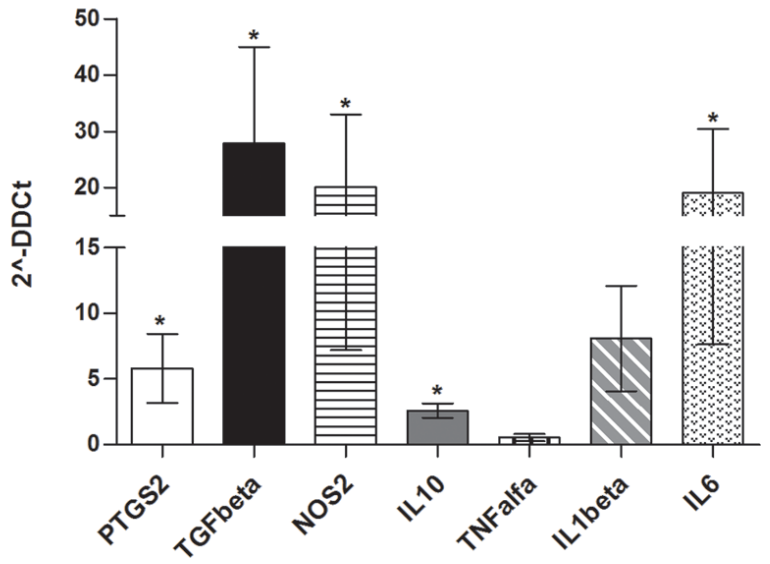

Figure 3: Expression of immune-modulatory factors by MM-MSC at Time 0 (A) and after 48 h of co-culture with PBMC (B). Only after incubation with PBMC, MM-MSCs showed statistically significant up-regulation of PTGS2, TGF $\beta$, NOS2, IL10 and IL6 expression $(p<0.05)$ compared with HD-MSCs (calculated value of $2^{\wedge}-\Delta \Delta C t$ in HD-MSC was 1 ). 


\section{DISCUSSION}

A well-recognized feature of $\mathrm{MM}$ is the intimate relationship between PC and BM microenvironment [4]. In this context, it is emerging the role of Myeloid-derived suppressor cells (MDSC) that are able to suppress immune responses, thus inducing an immunosuppressive milieu and contributing to create a permissive microenvironment that fosters evolution of disease [4, 11-17].

There are evidences that MDSC accumulation in $\mathrm{PB}$ and $\mathrm{BM}$ of $\mathrm{MM}$ patients correlates with disease progression and/or drug resistance [5], but little is known about the mechanisms of MDSC expansion, differentiation, and activation in MM. In this paper, we first confirmed that MDSC are increased in MM patients and are able to suppress T cells proliferation. Being MSC a key stromal cell population of the BM milieu that are able to transform the microenvironment into an immunosuppressive one in order to sustain PC proliferation, we have hypothesized the possibility that MSC could contribute to generate MDSC.

In fact, accumulating evidences indicate that tumor-associated microenvironment produces several factors involved in myelopoiesis and impairment of myeloid differentiation. A large amount of molecules released by tumor cells or tumor-surrounding cells, including IL- $1 \beta$, IL-4, IL-6, IL-10, IFN- $\gamma$ and TGF- $\beta$, are reported to re-program immature myeloid cells to become immunosuppressive $[5,36,40]$. Therefore, we investigated the direct role of MM-MSC in G-MDSC induction. Despite HD-, MGUS- and MM-MSC generate similar amount of G-MDSC, the ability to suppress T-lymphocytes proliferation was found only for G-MDSC that were generated after a co-culture with MSC derived from patients with MM and not from MGUS or healthy subjects. This finding is in line with observations from Sánchez et colleagues who showed that in a murine model oncogenesis drives MSC to increase the production of PGE2 favoring inhibition of lymphocytes proliferation and differentiation of myeloid precursors to highly suppressive cells [41]. However, one of the main question regarding MSC from MM patients stays still whether they are or not different from healthy MSC. Our work contribute to elucidate the different role of MM versus MGUS and healthy MSC, confirming the abnormal immune
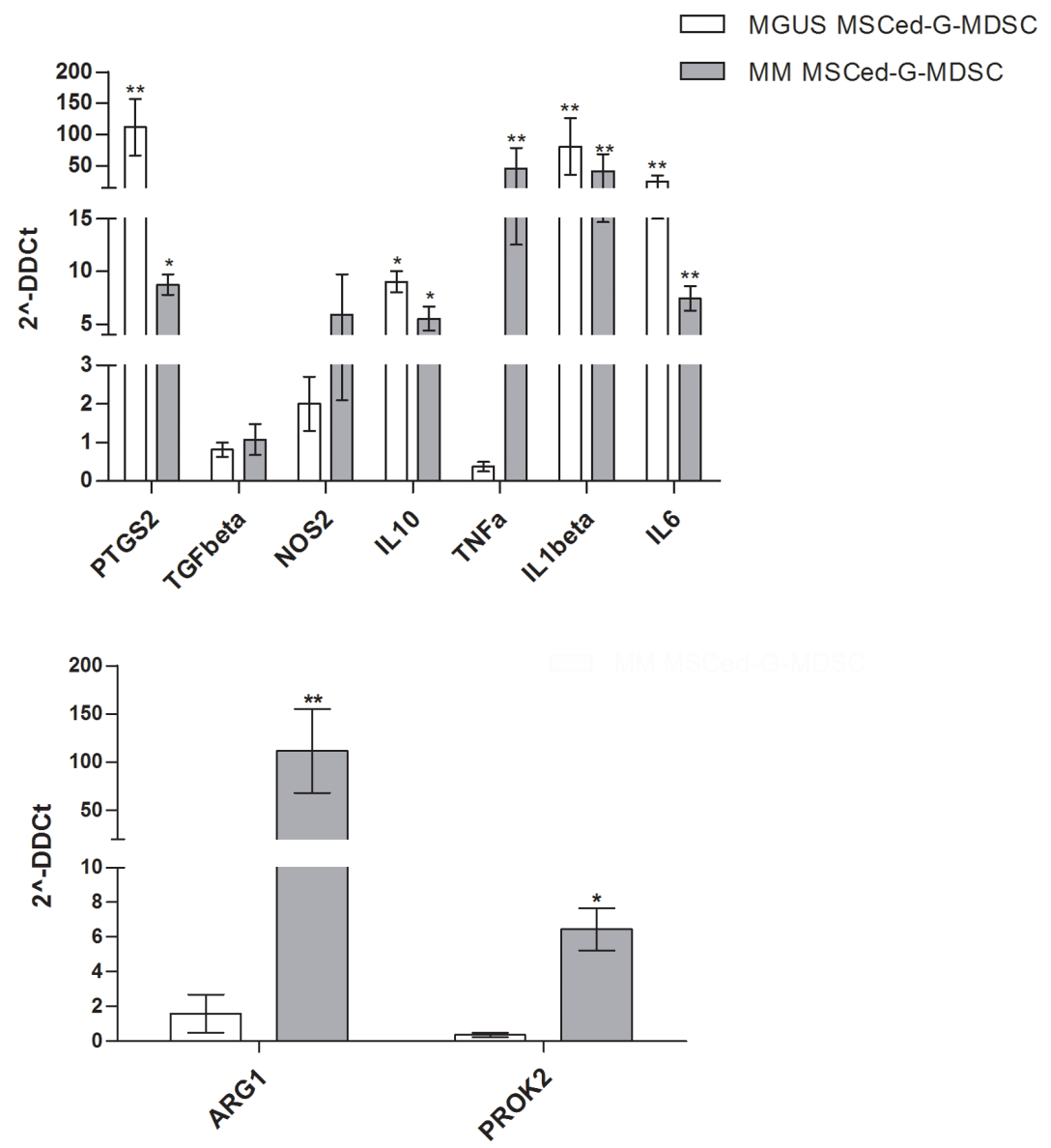

Figure 4: Expression of immune-modulatory and pro-angiogenic factors by MSCed-G-MDSC. The graphs report fold change values in gene expression of indicated genes (normalized to HD MSCed-G-MDSC) in MM- and MGUS-MSCed-G-MDSC. Only MM-MSCed-G-MDSC significantly upregulated TNFa, ARG1 and PROK2. Calculated value of $2^{\wedge}-\Delta \Delta \mathrm{Ct}$ in HD MSCed-G-MDSC was 1. 
modulatory ability of MM-MSC. Since this difference in G-MDSC activation has been found for isolated MM MSC after in vitro expansion, these stromal cells have a constitutive functional alteration in immune regulation.

When compared with their normal counterparts, MM-MSC differ in cytokine production, show a decreased proliferative ability with a premature senescence profile [42] and reduced capacity to inhibit $\mathrm{T}$ cell proliferation [43]. It is still an open question whether MSC have genomic alterations which may contribute to the pathogenesis of MM. MM-MSC exhibit a distinctive gene expression profile compared to HD-MSC [44], suggesting that these differences could be attributed to the presence of genomic alterations in MM counterpart $[45,46]$. Some reports have favored the possibility of a common haematopoietic and mesenchymal progenitor $[47,48]$. Garayoa et al. tried to find whether cytogenetic abnormalities present in PC were shared by matching MSC from the same patient [27]. All analyzed MMMSC were cytogenetically normal for the tested genomic alterations, thus excluding a common progenitor for MMPC and MSCs. With the present work, we suggest that MM-MSC can be functionally different from MSC from healthy subjects and MGUS, favoring immunosuppressive abilities of surrounding myeloid cells.
Exploring the immune-modulatory factors expressed by MM-MSC that are able to generate G-MDSC, we found a statistically significant up-regulation of PTGS2, TGF $\beta$, NOS2, IL10 and IL6 expression, suggesting that multiple mechanisms are involved in generation and activation of G-MDSC. Since gene expression changes were not found at $t 0$, the expression of the immune modulatory factors is influenced by interaction with PBMC in vitro, confirming that MM-MSC have constitutive immunological functional alterations.

Comparing MGUS- and MM-MSCed-G-MDSC to HD-MSCed-G-MDSC, both myeloid populations overexpressed IL-6, IL-1- $\beta$, PTGS2 and IL-10. However, only MM-MSCed-G-MDSC up-regulated TNF $\alpha$ and ARG1, providing thus evidence that MMMSC transform myeloid cells in immunosuppressive ones. In fact, up-regulation of ARG1 is one of the main mechanisms of MDSC-induced immunesuppression [49], while TNF $\alpha$ has been shown to arrest differentiation of immature myeloid cells and increase MDSC suppressive activity [50].

Yan et colleagues have also reported that IL-6 cooperates with G-CSF to induce tumor function of murine neutrophils in BM by modulating signaling pathways that favor tumor angiogenesis through
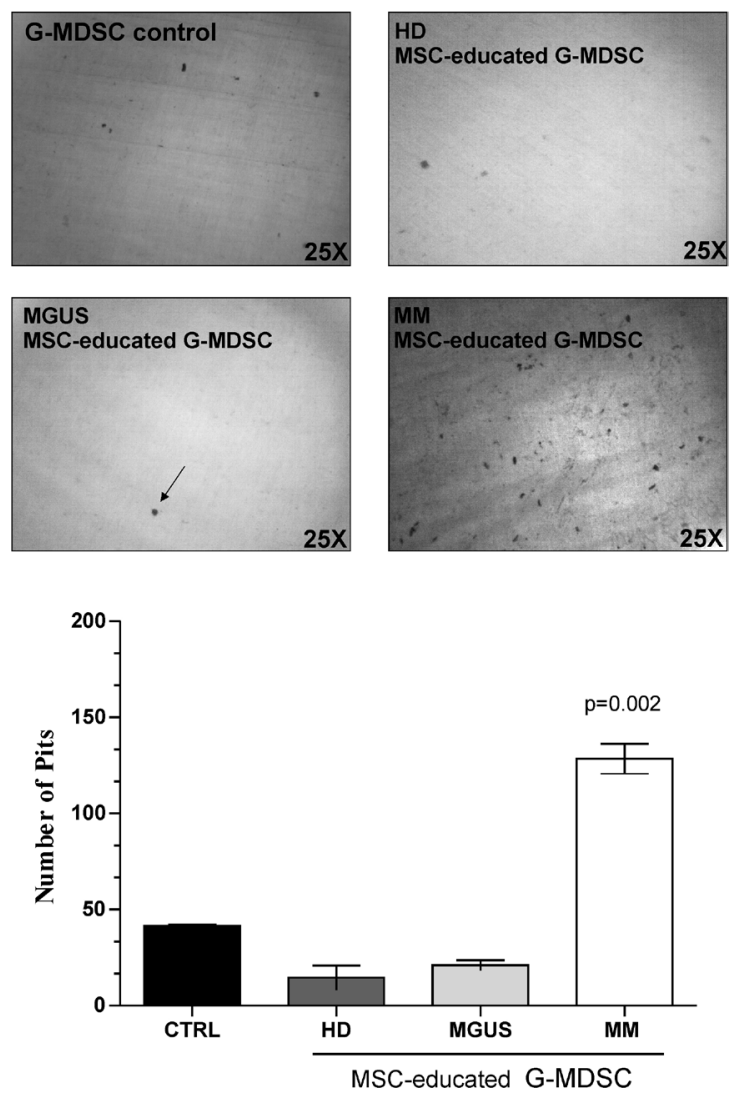

Figure 5: MM-MSCed-G-MDSC acquire bone resorption ability. Bone matrix digestion by MM-MSCed-G-MDSC is shown. The number of resorption pits was measured by light microscopy using Image $\mathrm{J}$ software. Results are expressed as the number of resorption pits per dentine disks (DDs). A significant digestive activity was observed only in DDs with MM MSCed-G-MDSC compared to G-MDSC control $(p=0.002)$. Values are shown as mean \pm SD of three independent experiments in duplicate. 
up-regulation of PROK2 [51]. Interestingly, we found the up-regulation of PROK2 by MM-MSCed-G-MDSC that may be linked to over-expression of IL6 by MM-MSC during co-culture with PBMC. PROK2 is a key regulator of inflammation-dependent tumorigenesis promoting chemotaxis, angiogenesis [38, 52] and drug-resistance into the tumor microenvironment [53]. Since MGUSMSCed-G-MDSC do not express PROK2, it is possible that MDSC present in MM patients may contribute to the "angiogenic switch" that characterizes the transition from MGUS to MM.

The osteoblasts derived from MM-MSC exhibit a diminished matrix mineralization potential when compared with MSC from healthy donors [44]. Furthermore, the clinical observation that bone lesions persist in patients who respond to therapy supports the idea of a permanent defect in the MM-MSC [54]. Previous studies have shown that in response to $\mathrm{PC}, \mathrm{MM}$ associated-mesenchyme release pro-osteoclastogenic cytokines that increase osteoclasts (OC) recruitment and OC-mediated bone loss $[55,56]$. STRO-1 is a well characterized MSC antigen expressed by a population of immature and multipotent MSC [57]. Noll et al. demonstrated increased amount of STRO-1 MSC in MM patients that correlated with more severe disease and that expressed higher levels of PC- and OC-activating factors, including RANKL and IL6 [55]. Recently, it has been reported that neutrophils can acquire monocytic characteristics in response to inflammatory signals [58]. For the first time, we demonstrated that, unlike MGUS-, MM-MSCed-G-MDSC were able to digest bone matrix, suggesting that MM-MSC may indirectly contribute to bone resorption by G-MDSC activation.

In conclusion, these findings strongly support an evolving concept regarding the contribution of MM-MSC in tumor development and progression, indicating that cancer progression may rely on MSC both directly and through G-MDSC-mediated immunosuppression into the MM microenvironment, leading to the cancer immune surveillance evasion. The acquisition by MM-MSC of the ability to induce G-MDSC with an immunosuppressive behavior and digestive ability might represent an evolutionary advantage acquired during the multistep development of cancer.

Taken together, our data give further evidence of the key role played by MSC in MM BM microenvironment, making it an immune-tolerant milieu through G-MDSC induction. Therefore, the interaction of MM cells with MSC creates an important loop: PC stimulate the proliferation of MSC [55] and influence their gene expression profile [59], but at the same time MSC support MM cell growth and survival by releasing several cytokines and growth factors, and by promoting MDSC activation (Figure 6). Elucidation of the complex interactions between MM cells and MSC into MM

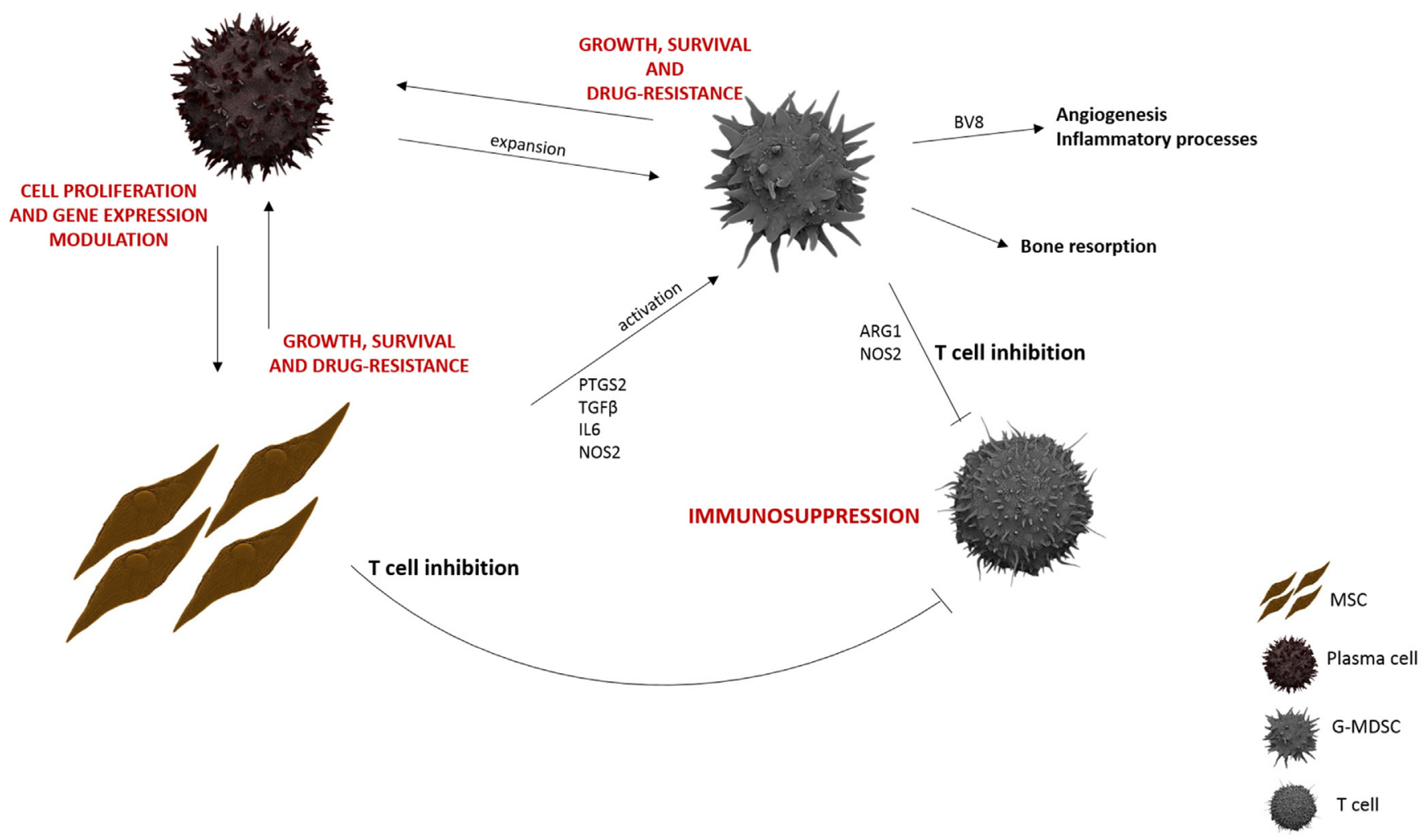

Figure 6: MM BM microenvironment. MM-MSC, both directly and through G-MDSC activation, support MM growth, survival and drug-resistance within an immunosuppressive BM microenvironment. 
microenvironment is necessary in the development of effective therapies to improve treatment of MM.

\section{MATERIALS AND METHODS}

\section{Patients and sample collection}

After written informed consent approved by the local ethical committee (Azienda ospedaliero Universitaria Policlinico-Vittorio Emanuele, \# 34/2013/VE), samples were collected from MGUS $(n=30)$, newly diagnosed $(n=30)$ and relapsedMM $(n=15)$ patients and age-matched HD $(n=30)$ at Division of Hematology, AOU Policlinico-OVE, University of Catania. Clinical data of MM and MGUS patients included in this study are shown in Table 1. Patients were free of immune-mediated diseases and acute or chronic viral infections to exclude any interference on immune-regulatory mechanisms. All MGUS patients had a stable condition with at least 2 years of follow up.

\section{G-MDSC evaluation}

Whole blood collected in EDTA vials $(50 \mu \mathrm{L})$ was stained with monoclonal antibodies (moAbs, $10 \mu \mathrm{L}$ for each) and respective isotypic controls [34]. The moAbs (Beckman coulter) included: CD11b FITC (clone bear-1), CD15 PE (clone 80H5), CD14 PC5, (clone RMO52), HLA-DR- ECD (Clone Immu-357). Using sequential gating strategy, G-MDSC were identified

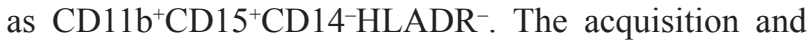
analysis was performed with a Beckman Coulter FC-500 flow cytometer (10,000 cells were analyzed).

To evaluate the suppressive ability, G-MDSC from MM patients and HD were isolated using antiCD66 magnetic microbeads (Miltenyi Biotec) and then co-cultured for three days with autologous Carboxyfluorescein succinimidyl ester (CFSE)-labeled $\mathrm{T}$ lymphocytes at ratio 1:4 [35]. For cell labeling, $5 \times 10^{5}$ lymphocytes were incubated at $37^{\circ} \mathrm{C}$ for $20 \mathrm{~min}$ in $1 \mathrm{ml}$ PBS containing $1 \mu \mathrm{M}$ CFSE (BD Pharmingen). T cells were stimulated with $5 \mathrm{mg} / \mathrm{mL}$ phytohemagglutinin (PHA) and incubated for 72 hours prior to flow cytometry. Controls included a positive $\mathrm{T}$ cell proliferation control ( $\mathrm{T}$ cells plus PHA) and a negative one ( $\mathrm{T}$ cells only). After three days, $\mathrm{T}$ cell proliferation was measured by CFSE dilution and analyzed using flow cytometry.

\section{MSC harvest and culture}

BM mononuclear cells from HD $(n=6)$, MGUS $(n=5)$ and MM $(n=7,4$ of which at diagnosis and 3 relapsed) subjects were obtained after density gradient centrifugation on Ficoll and cultured in low-glucose Dulbecco's modified Eagle's medium supplemented with $10 \%$ heat-inactivated FBS, $100 \mathrm{U} / \mathrm{ml}$ penicillin, $100 \mathrm{mg} / \mathrm{ml}$ streptomycin and 1\% L-glutamine. After 3 days in culture, non-adherent cells were removed, whereas MSCs were selected by their adherence to the plastic-ware. The cultures were maintained at $37^{\circ} \mathrm{C}$ and $5 \% \mathrm{CO}_{2}$. MSCs were expanded until the third or fourth passage and then trypsinized to be used for experiments.

Selected MSC from both patients and HD at the third passage were also tested for MSC specific surface antigen expression (Supplementary Figure 3). Therefore, cells were labeled using combinations of monoclonal antibodies: anti-CD34-ECD (clone 581), anti-CD90FITC (clone F15.42.1.5), anti-CD105-PE (clone 1G2) and anti-CD45-PC5 (clone J.33). The appropriate isotopic control was also included. Labeled MSC were acquired using a Beckman Coulter FC-500 flow cytometer.

\section{MDSC induction}

Human peripheral blood mononucleated cells (PBMC) were isolated from healthy volunteer donors after density gradient centrifugation on Ficoll. PBMC were cultured alone or co-cultured with MSC derived from healthy, MGUS or MM subjects as described above (1:100 ratio). MSC were seeded to achieve confluence by 7 days. After one week, PBMC were collected and G-MDSCs were isolated using anti-CD66b magnetic microbeads. The phenotype of G-MDSC was confirmed by cytofluorimetric analysis. Their immunosuppressive capacity was analyzed by evaluating $\mathrm{T}$ cell anergy when co-cultured with autologous CFSE-labeled T cells stimulated by PHA. Controls included a positive $\mathrm{T}$ cell proliferation control ( $\mathrm{T}$ cells plus PHA) and a negative one ( $\mathrm{T}$ cells only). After three days $\mathrm{T}$ cell proliferation was analyzed by flow cytometry.

\section{Real-time RT-PCR for gene expression of MSC and MDSC}

For gene expression studies, MSC were trypsinized from culture flasks both at Time 0 (cells at confluence incubated with standard medium only) and after 48 hours from start of co-culture experiments. In addition, MSC co-cultured with PBMC were purified using anti-CD271 magnetic microbeads. G-MDSC control (isolated from PBMC cultured in medium alone), MGUSand MM-MSC-educated G-MDSC were collected from co-cultures and isolated by magnetic microbeads. After RNA extraction and reverse transcription, we evaluated expression of the following mRNA: ARG1 (Arginase 1), NOS2 (Nitric Oxide Synthase 2), PTGS2 (Prostaglandin-Endoperoxide Synthase 2), TNF $\alpha$ (Tumor necrosis factor alpha), TGF $\beta$ (Transforming growth factor beta), IL6 (Interleukin 6), IL10 (Interleukin 10), IL1 $\beta$ (Interleukin 1beta), and PROK2 (Prokineticin 2/BV8). Their expression was assessed by TaqMan Gene Expression (Life Technologies) and quantified using a fluorescence-based real-time detection 
Table 1: Baseline clinical characteristics of patients included in the study

\begin{tabular}{|c|c|c|c|}
\hline & $\begin{array}{l}\text { MGUS } \\
(n=30)\end{array}$ & $\begin{array}{c}\text { Newly-diagnosed } \\
\text { MM }(n=30)\end{array}$ & $\begin{array}{c}\text { Relapsed } \\
\text { MM }(N=15)\end{array}$ \\
\hline Median age (range) & $67(49-70)$ & $64(40-81)$ & $67(38-75)$ \\
\hline Males/Females & $21 / 9$ & $17 / 13$ & $8 / 7$ \\
\hline \multicolumn{4}{|l|}{ Istotype, $n$} \\
\hline $\operatorname{Ig} G$ & 0 & 16 & 6 \\
\hline $\operatorname{Ig} A$ & 6 & 7 & 5 \\
\hline Light-chain only & 0 & 7 & 4 \\
\hline \multicolumn{4}{|l|}{ Cytogenetics, $n$} \\
\hline Normal & 15 & 12 & 1 \\
\hline del 13 & 2 & 5 & 4 \\
\hline del 17 & 0 & 6 & 4 \\
\hline$t(4 ; 14)$ & 3 & 3 & 4 \\
\hline not performed/failed & 10 & 4 & 2 \\
\hline Haemoglobin, g/dl (range) & $12.8(12-14.5)$ & $10.6(6.5-13.8)$ & $9.8(6.6-12.8)$ \\
\hline Platelets $1000 / u L$ (range) & $219(180-315)$ & $221(90-384)$ & $123(43-225)$ \\
\hline Bone marrow plasmocytosis $>50 \%, n(\%)$ & 0 & 12 & 12 \\
\hline C-reactive protein median, mg/l (range) & $0.1(0.01-4)$ & $4.4(0.01-8.5)$ & $5.3(0.05-9.6)$ \\
\hline LDH median, U/l (range) & $195(132-213)$ & $209(109-708)$ & $240(125-368)$ \\
\hline ESR median, mm/h & $17(0-26)$ & $72(6-134)$ & $84(10-138)$ \\
\hline \multicolumn{4}{|l|}{ STAGE ISS, n } \\
\hline 1 & N.A. & 7 & 0 \\
\hline 2 & N.A. & 15 & 10 \\
\hline 3 & N.A. & 8 & 5 \\
\hline
\end{tabular}

WBC: white blood cells count; ALC: absolute lymphocyte count; ESR: Erythrocyte Sedimentation Rate; ISS: International Staging System.

method by 7900HT Fast Start (Life Technologies). For each sample, the relative expression level of each studied mRNA was normalized using GAPDH as invariant controls.

\section{Resorption assay}

MSC-educated G-MDSC $\left(2 \times 10^{4} / \mathrm{cm}^{2}\right)$ were plated onto dentine discs (Osteosite Dentine Discs (DDs), Immunodiagnostic Systems Inc., Fountain Hills, AR) in 96-well plates for the digestion test. After $96 \mathrm{~h}$, the cells were removed with 5\% sodium hypochlorite for $10 \mathrm{~min}$. Discs were rinsed with water and stained with $1 \%(\mathrm{w} / \mathrm{v})$ toluidine blue in $0.5 \%$ sodium borate for $30 \mathrm{~s}$ and then washed twice with water. The number and the area of resorption pits were then measured by light microscopy using ImageJ software. Results were expressed as the number of resorption pits. Experiments were performed in triplicate.

\section{Statistical analysis}

Statistical analyses were made with Prism Software (Graphpad Software Inc., La Jolla, CA, USA). Data were expressed as mean or SD. Statistical analysis was carried out by unpaired $t$-test or ANOVA test. A $p$-value of 0.05 was considered to indicate a statistically significant difference between experimental and control groups.

\section{ACKNOWLEDGMENTS}

This study was supported in part by AIL (Associazione Italiana contro le Leucemie) sezione di Catania, FON.CA.NE.SA. (Fondazione Catanese per lo Studio delle Malattie Neoplastiche del Sangue). 


\section{GRANT SUPPORT}

Financial support (Prof. Francesco Di Raimondo) Ministero della Salute, Ricerca Finalizzata 2011-2012 (PE-2011-02350147).

\section{CONFLICTS OF INTEREST}

The authors disclose no potential conflicts of interest.

\section{REFERENCES}

1. Pfreundschuh M. Inheritance in MGUS and MM. Oncotarget. 2015; 6:32287-8. doi: 10.18632/oncotarget.5960.

2. Guglielmelli T, Giugliano E, Brunetto V, Rapa I, Cappia S, Giorcelli J, Rrodhe S, Papotti M, Saglio G. mTOR pathway activation in multiple myeloma cell lines and primary tumour cells: pomalidomide enhances cytoplasmic-nuclear shuttling of mTOR protein. Oncoscience. 2015; 2:382-394. doi: 10.18632/oncoscience.148.

3. Tibullo D, Di Rosa M, Giallongo C, La Cava P, Parrinello NL, Romano A, Conticello C, Brundo MV, Saccone S, Malaguarnera L, Di Raimondo F. Bortezomib modulates CHIT1 and YKL40 in monocyte-derived osteoclast and in myeloma cells. Frontiers in pharmacology. 2015; 6:226.

4. Romano A, Conticello C, Cavalli M, Vetro C, La Fauci A, Parrinello NL, Di Raimondo F. Immunological dysregulation in multiple myeloma microenvironment. BioMed research international. 2014; 2014:198539.

5. Botta C, Gulla A, Correale P, Tagliaferri P, Tassone P. Myeloid-derived suppressor cells in multiple myeloma: preclinical research and translational opportunities. Frontiers in oncology. 2014; 4:348.

6. Wang J, De Veirman K, De Beule N, Maes K, De Bruyne E, Van Valckenborgh E, Vanderkerken K, Menu E. The bone marrow microenvironment enhances multiple myeloma progression by exosome-mediated activation of myeloidderived suppressor cells. Oncotarget. 2015; 6:43992-44004. doi: 10.18632/oncotarget.6083.

7. Hu J, Van Valckenborgh E, Menu E, De Bruyne E, Vanderkerken K. Understanding the hypoxic niche of multiple myeloma: therapeutic implications and contributions of mouse models. Disease models and mechanisms. 2012; 5:763-771.

8. Wang L, Wang H, Chen H, Wang WD, Chen XQ, Geng QR, Xia ZJ, Lu Y. Serum levels of soluble programmed death ligand 1 predict treatment response and progression free survival in multiple myeloma. Oncotarget. 2015; 6: 41228-41236. doi: 10.18632/oncotarget.5682.

9. Gabrilovich DI, Nagaraj S. Myeloid-derived suppressor cells as regulators of the immune system. Nature reviews Immunology. 2009; 9:162-174.

10. Montero AJ, Diaz-Montero CM, Kyriakopoulos CE, Bronte V, Mandruzzato S. Myeloid-derived suppressor cells in cancer patients: a clinical perspective. J Immunother. 2012; 35:107-115.

11. Chen S, Zhang Y, Zhang B. MicroRNA-155 regulates tumor myeloid-derived suppressive cells. Oncoscience. 2015; 2:910-911. doi: 10.18632/oncoscience.269.

12. Gorgun GT, Whitehill G, Anderson JL, Hideshima T, Maguire C, Laubach J, Raje N, Munshi NC, Richardson PG, Anderson KC. Tumor-promoting immune-suppressive myeloid-derived suppressor cells in the multiple myeloma microenvironment in humans. Blood. 2013; 121: 2975-2987.

13. Favaloro J, Liyadipitiya T, Brown R, Yang S, Suen H, Woodland N, Nassif N, Hart D, Fromm P, Weatherburn C, Gibson J, Ho PJ, Joshua D. Myeloid derived suppressor cells are numerically, functionally and phenotypically different in patients with multiple myeloma. Leuk Lymphoma. 2014; 55:2893-2900.

14. Ramachandran IR, Martner A, Pisklakova A, Condamine T, Chase T, Vogl T, Roth J, Gabrilovich D, Nefedova Y. Myeloid-derived suppressor cells regulate growth of multiple myeloma by inhibiting $\mathrm{T}$ cells in bone marrow. Journal of immunology. 2013; 190:3815-3823.

15. Wang Z, Zhang L, Wang H, Xiong S, Li Y, Tao Q, Xiao W, Qin H, Wang Y, Zhai Z. Tumor-induced CD14 (+) HLADR (-/low) myeloid-derived suppressor cells correlate with tumor progression and outcome of therapy in multiple myeloma patients. Cancer Immunol Immunother. 2015; 64:389-399.

16. De Veirman K, Van Valckenborgh E, Lahmar Q, Geeraerts X, De Bruyne E, Menu E, Van Riet I, Vanderkerken K, Van Ginderachter JA. Myeloid-derived suppressor cells as therapeutic target in hematological malignancies. Frontiers in oncology. 2014; 4:349.

17. Van Valckenborgh E, Schouppe E, Movahedi K, De Bruyne E, Menu E, De Baetselier P, Vanderkerken K, Van Ginderachter JA. Multiple myeloma induces the immunosuppressive capacity of distinct myeloid-derived suppressor cell subpopulations in the bone marrow. Leukemia. 2012; 26:2424-2428.

18. De Veirman K, Van Ginderachter JA, Lub S, De Beule N, Thielemans K, Bautmans I, Oyajobi BO, De Bruyne E, Menu E, Lemaire M, Van Riet I, Vanderkerken K, Van Valckenborgh E. Multiple myeloma induces Mcl-1 expression and survival of myeloid-derived suppressor cells. Oncotarget. 2015; 6:10532-10547. doi: 10.18632/oncotarget.3300.

19. Brandau S, Trellakis S, Bruderek K, Schmaltz D, Steller G, Elian M, Suttmann H, Schenck M, Welling J, Zabel P, Lang S. Myeloid-derived suppressor cells in the peripheral blood of cancer patients contain a subset of immature neutrophils with impaired migratory properties. Journal of leukocyte biology. 2011; 89:311-317.

20. Nagaraj S, Gabrilovich DI. Regulation of suppressive function of myeloid-derived suppressor cells by $\mathrm{CD} 4+\mathrm{T}$ cells. Semin Cancer Biol. 2012; 22:282-288. 
21. Movahedi K, Guilliams M, Van den Bossche J, Van den Bergh R, Gysemans C, Beschin A, De Baetselier P, Van Ginderachter JA. Identification of discrete tumor-induced myeloid-derived suppressor cell subpopulations with distinct $\mathrm{T}$ cell-suppressive activity. Blood. 2008; 111: 4233-4244.

22. Nagaraj S, Schrum AG, Cho HI, Celis E, Gabrilovich DI. Mechanism of $\mathrm{T}$ cell tolerance induced by myeloid-derived suppressor cells. Journal of immunology (Baltimore, Md : 1950). 2010; 184:3106-3116.

23. Sawant A, Ponnazhagan S. Myeloid-derived suppressor cells as osteoclast progenitors: a novel target for controlling osteolytic bone metastasis. Cancer research. 2013; 73: 4606-4610.

24. Sawant A, Ponnazhagan S. Myeloid-derived suppressor cells as a novel target for the control of osteolytic bone disease. Oncoimmunology. 2013; 2:e24064.

25. Zhuang J, Zhang J, Lwin ST, Edwards JR, Edwards CM, Mundy GR, Yang X. Osteoclasts in multiple myeloma are derived from Gr-1+CD11b+myeloid-derived suppressor cells. PloS one. 2012; 7:e48871.

26. Minguell JJ, Erices A, Conget P. Mesenchymal stem cells. Experimental biology and medicine. 2001; 226:507-520.

27. Garayoa M, Garcia JL, Santamaria C, Garcia-Gomez A, Blanco JF, Pandiella A, Hernandez JM, Sanchez-Guijo FM, del Canizo MC, Gutierrez NC, San Miguel JF. Mesenchymal stem cells from multiple myeloma patients display distinct genomic profile as compared with those from normal donors. Leukemia. 2009; 23:1515-1527.

28. Uccelli A, Moretta L, Pistoia V. Mesenchymal stem cells in health and disease. Nat Rev Immunol. 2008; 8:726-736.

29. Poggi A, Musso A, Dapino I, Zocchi MR. Mechanisms of tumor escape from immune system: role of mesenchymal stromal cells. Immunology letters. 2014; 159:55-72.

30. Nauta AJ, Fibbe WE. Immunomodulatory properties of mesenchymal stromal cells. Blood. 2007; 110:3499-3506.

31. Mougiakakos D, Jitschin R, Johansson CC, Okita R, Kiessling R, Le Blanc K. The impact of inflammatory licensing on heme oxygenase-1-mediated induction of regulatory $\mathrm{T}$ cells by human mesenchymal stem cells. Blood. 2011; 117:4826-4835.

32. Soleymaninejadian E, Pramanik K, Samadian E. Immunomodulatory properties of mesenchymal stem cells: cytokines and factors. American journal of reproductive immunology. 2012; 67:1-8.

33. Wang G, Zhang S, Wang F, Li G, Zhang L, Luan X. Expression and biological function of programmed death ligands in human placenta mesenchymal stem cells. Cell biology international. 2013; 37:137-148.

34. Duffy A, Zhao F, Haile L, Gamrekelashvili J, Fioravanti S, Ma C, Kapanadze T, Compton K, Figg WD, Greten TF. Comparative analysis of monocytic and granulocytic myeloid-derived suppressor cell subsets in patients with gastrointestinal malignancies. Cancer immunology, immunotherapy : CII. 2013; 62:299-307.

35. Giallongo C, Parrinello N, Tibullo D, La Cava P, Romano A, Chiarenza A, Barbagallo I, Palumbo GA, Stagno F, Vigneri P, Di Raimondo F. Myeloid derived suppressor cells (MDSCs) are increased and exert immunosuppressive activity together with polymorphonuclear leukocytes (PMNs) in chronic myeloid leukemia patients. PloS one. 2014; 9:e101848.

36. Gabrilovich DI, Ostrand-Rosenberg S, Bronte V. Coordinated regulation of myeloid cells by tumours. Nature reviews Immunology. 2012; 12:253-268.

37. LeCouter J, Ferrara N. EG-VEGF and Bv8. a novel family of tissue-selective mediators of angiogenesis, endothelial phenotype, and function. Trends in cardiovascular medicine. 2003; 13:276-282.

38. Qu X, Zhuang G, Yu L, Meng G, Ferrara N. Induction of Bv8 expression by granulocyte colony-stimulating factor in $\mathrm{CD} 11 \mathrm{~b}+\mathrm{Gr} 1+$ cells: key role of Stat 3 signaling. The Journal of biological chemistry. 2012; 287:19574-19584.

39. Munder M. Arginase: an emerging key player in the mammalian immune system. British journal of pharmacology. 2009; 158:638-651.

40. Gallina G, Dolcetti L, Serafini P, De Santo C, Marigo I, Colombo MP, Basso G, Brombacher F, Borrello I, Zanovello P, Bicciato S, Bronte V. Tumors induce a subset of inflammatory monocytes with immunosuppressive activity on $\mathrm{CD} 8+\mathrm{T}$ cells. The Journal of clinical investigation. 2006; 116:2777-2790.

41. Sanchez N, Miranda A, Funes JM, Hevia G, Perez R, de Leon J. Oncogenic transformation tunes the cross-talk between mesenchymal stem cells and $\mathrm{T}$ lymphocytes. Cellular immunology. 2014; 289:174-184.

42. Andre T, Meuleman N, Stamatopoulos B, De Bruyn C, Pieters K, Bron D, Lagneaux L. Evidences of early senescence in multiple myeloma bone marrow mesenchymal stromal cells. PloS one. 2013; 8:e59756.

43. Li B, Fu J, Chen $\mathrm{P}$, Zhuang W. Impairment in immunomodulatory function of mesenchymal stem cells from multiple myeloma patients. Archives of medical research. 2010; 41:623-633.

44. Corre J, Mahtouk K, Attal M, Gadelorge M, Huynh A, Fleury-Cappellesso S, Danho C, Laharrague P, Klein B, Reme T, Bourin P. Bone marrow mesenchymal stem cells are abnormal in multiple myeloma. Leukemia. 2007; 21:1079-1088.

45. Garderet L, Mazurier C, Chapel A, Ernou I, Boutin L, Holy X, Gorin NC, Lopez M, Doucet C, Lataillade JJ. Mesenchymal stem cell abnormalities in patients with multiple myeloma. Leuk Lymphoma. 2007; 48:2032-2041.

46. Arnulf B, Lecourt S, Soulier J, Ternaux B, Lacassagne MN, Crinquette A, Dessoly J, Sciaini AK, Benbunan M, Chomienne C, Fermand JP, Marolleau JP, Larghero J. Phenotypic and functional characterization of bone marrow 
mesenchymal stem cells derived from patients with multiple myeloma. Leukemia. 2007; 21:158-163.

47. Zhang W, Knieling G, Vohwinkel G, Martinez T, Kuse R, Hossfeld DK, Duhrsen U. Origin of stroma cells in longterm bone marrow cultures from patients with acute myeloid leukemia. Annals of hematology. 1999; 78:305-314.

48. Huss R, Moosmann S. The co-expression of CD117 (c-kit) and osteocalcin in activated bone marrow stem cells in different diseases. Br J Haematol. 2002; 118:305-312.

49. Giallongo C, Parrinello N, Brundo MV, Raccuia SA, Di Rosa M, La Cava P, Tibullo D. Myeloid derived suppressor cells in chronic myeloid leukemia. Frontiers in oncology. 2015; 5:107.

50. Sade-Feldman M, Kanterman J, Ish-Shalom E, Elnekave M, Horwitz E, Baniyash M. Tumor necrosis factor-alpha blocks differentiation and enhances suppressive activity of immature myeloid cells during chronic inflammation. Immunity. 2013; 38:541-554.

51. Yan B, Wei JJ, Yuan Y, Sun R, Li D, Luo J, Liao SJ, Zhou YH, Shu Y, Wang Q, Zhang GM, Feng ZH. IL-6 cooperates with G-CSF to induce protumor function of neutrophils in bone marrow by enhancing STAT3 activation. Journal of immunology. 2013; 190:5882-5893.

52. Ferrara N. Role of myeloid cells in vascular endothelial growth factor-independent tumor angiogenesis. Current opinion in hematology. 2010; 17:219-224.

53. Chung AS, Wu X, Zhuang G, Ngu H, Kasman I, Zhang J, Vernes JM, Jiang Z, Meng YG, Peale FV, Ouyang W, Ferrara N. An interleukin-17-mediated paracrine network promotes tumor resistance to anti-angiogenic therapy. Nature medicine. 2013; 19:1114-1123.

54. Terpos E, Heath DJ, Rahemtulla A, Zervas K, Chantry A, Anagnostopoulos A, Pouli A, Katodritou E, Verrou E,
Vervessou EC, Dimopoulos MA, Croucher PI. Bortezomib reduces serum dickkopf-1 and receptor activator of nuclear factor-kappaB ligand concentrations and normalises indices of bone remodelling in patients with relapsed multiple myeloma. British journal of haematology. 2006; 135:688-692.

55. Noll JE, Williams SA, Tong CM, Wang H, Quach JM, Purton LE, Pilkington K, To LB, Evdokiou A, Gronthos S, Zannettino AC. Myeloma plasma cells alter the bone marrow microenvironment by stimulating the proliferation of mesenchymal stromal cells. Haematologica. 2014; 99:163-171.

56. Zannettino AC, Farrugia AN, Kortesidis A, Manavis J, To LB, Martin SK, Diamond P, Tamamura H, Lapidot T, Fujii N, Gronthos S. Elevated serum levels of stromalderived factor-1alpha are associated with increased osteoclast activity and osteolytic bone disease in multiple myeloma patients. Cancer research. 2005; 65:1700-1709.

57. Simmons PJ, Torok-Storb B. Identification of stromal cell precursors in human bone marrow by a novel monoclonal antibody, STRO-1. Blood. 1991; 78:55-62.

58. Koffel R, Meshcheryakova A, Warszawska J, Hennig A, Wagner K, Jorgl A, Gubi D, Moser D, Hladik A, Hoffmann U, Fischer MB, van den Berg W, Koenders M, et al. Monocytic cell differentiation from band-stage neutrophils under inflammatory conditions via MKK6 activation. Blood. 2014; 124:2713-2724.

59. Garcia-Gomez A, De Las Rivas J, Ocio EM, Diaz-Rodriguez E, Montero JC, Martin M, Blanco JF, Sanchez-Guijo FM, Pandiella A, San Miguel JF, Garayoa M. Transcriptomic profile induced in bone marrow mesenchymal stromal cells after interaction with multiple myeloma cells: implications in myeloma progression and myeloma bone disease. Oncotarget. 2014; 5:8284-8305. doi: 10.18632/oncotarget.2058. 